\title{
Pseudo-Data Generation Software for FBG-Optical Fiber Based Shape Sensing
}

\author{
Hendrikje Pauer ${ }^{1}$, Christoph Ledermann ${ }^{1}$, Wilderich Tuschmann ${ }^{2}$, Heinz Wörn ${ }^{1}$ \\ Karlsruhe Institute of Technology - KIT \\ Institute for Anthropomatics and Robotics (IAR) - Intelligent Process Control and Robotics (IPR) ${ }^{1}$ \\ Institute for Algebra and Geometry (IAG) - Research Group Differential Geometry ${ }^{2}$ \\ Engler-Bunte-Ring 8, 76131 Karlsruhe, Germany \\ hendrikje.pauer@kit.edu
}

\begin{abstract}
Shape sensing based on FBG-optical fibers is a current research topic of several working groups worldwide. The sensor is integrated into flexible snakelike objects such as e.g. endoscopes or flexible tubes to detect shape deformation. This way, the objects can be observed and tracked. Shapesensing supports navigation of flexible instruments. Currently, robotic systems consist of ridgit joints only. Shapesensing provides the required information for automated control of the robot end-effector position and orientation in case additional flexible joints are integrated into the robotic system. In this field of research, some fundamental aspects have already been researched and standard algorithms are published. As the sensor technology is supposed to be used in medical technology, the requirements towards the sensor accuracy and robustness are demanding. Therefor, shapesensing still needs to be improved. At the Institute for Anthropomatics and Robotics Intelligent Process Control and Robotics - Karlsruhe Institute of Technology (IAR-IPR-KIT), fundamentally new sensor concepts are investigated. However, the testing of new algorithms and sensor designs is very costly as a high number of prototypes has to be build. To avoid these costs in time and money, the tests are planned based on pseudo measurement data. To efficiently generate this kind of data, we developed a software. In this paper, the individual parts of the software are presented and the main functionality and easy-of-use are evaluated.
\end{abstract}

\section{Introduction and State of the Art}

FBG-optical fiber based shape sensing has gained more and more importance in recent years. A shape sensor usually consists of a flexible snakelike carrier body with embedded fiber-Bragg grating optical glass fibers. The fibers detect strain that occurs in the carrier material caused by bending of the sensor body. Through application of specific algorithms to this measurement data, the sensor shape can be reconstructed. By guiding the sensor along flexible instruments as e.g. endoscopes, the instrument's shape, its tip position and orientation can be tracked.

Several working groups have achieved fundamental results on this area. Algorithms for shape reconstruction are given by [1] and provide one basic solution for shapesensoring. The way of building the sensor is implemented in various ways. Different kinds of material are used as a carrier body. Also the way of fiber guidance differs. [2], [3], [4], [5], [6], [7], [8], [4]

The Institute for Anthropomatics and Robotics - Intelligent Process Control and Robotics - Karlsruhe Institute of Technology (IAR-IPR-KIT) is doing research on shape sensing since 2011. The standard algorithms for shape reconstruction are implemented and various prototypes of different material are build to test the main functionalities of the sensor system [9], [10], [11], [12]. One prototype is shown in Fig. 1.

At the IAR-IPR-KIT, shape sensing is developed to be used in medical technology as this is one major field of applica-

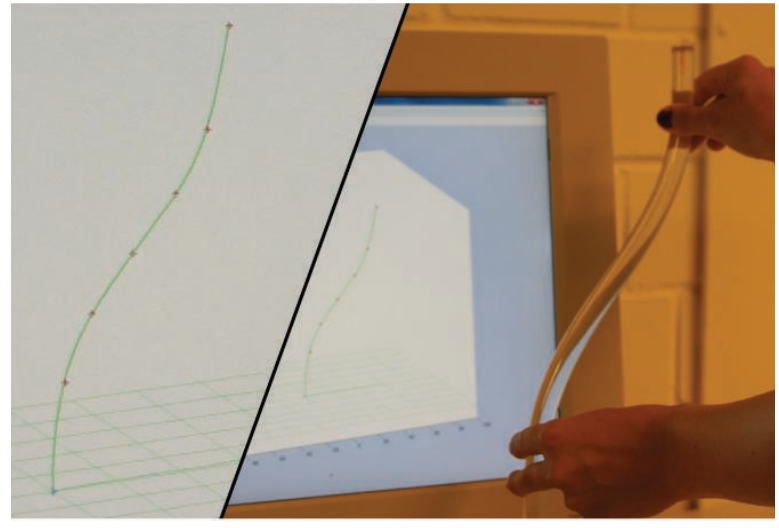

Abbildung 1 IPR prototype of a shapesensor

tion for that kind of sensor. The sensor can be used to facilitate and improve the navigation of flexible instruments during minimally invasive surgery. Furthermore, the sensor information allows automated robot-assisted surgery even in case where not only, as currently usual, rigid robot joints, but also flexible parts are integrated into the robotic system. As the sensor is supposed to be used in minimally invasive surgery, the requirements for sensor precision are demanding. In this regard, the research group at the KIT aims to continually improve the sensor quality, enhancing precision and reliability.

For further improvements of shape sensing in order to 


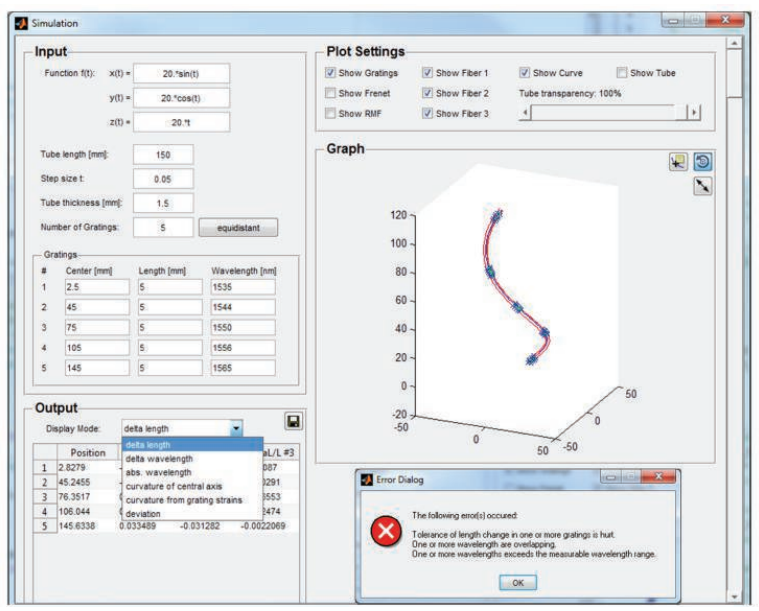

Abbildung 2 Previous IPR software-solution for pseudo data generation

achieve sufficiently high sensor accuracy, fundamentally new concepts of shape sensing are developed. The algorithms and the way of fiber distribution along the sensor carrier body are supposed to be approached in a fundamentally new and redesigned way. Therefore, a large number of different sensor model variations have to be tested. To enable efficient research and development of new sensor configurations, it is helpful to use pseudo-measurement data for testing purpose. This way, the basic functionality of new sensor configurations and reconstruction algorithms can be pre-evaluated before producing a prototype, which involves considerable time and expense of material costs. One additional advantage of using pseudo-data is as follows: The pseudo measurement data is generated based on a given sensor deformation. Therewith, a ground truth for the shape that has to be reconstructed by the sensor is already known. The shape reconstruction result can be compared with this given shape and the sensor accuracy can be easily evaluated. Using real data, it always constitutes significant difficulties to get reference values for the present shape.

Testing based on pseudo-measurement data is a common method in technical research and already established in many fields of application. Though, to the best of the authors knowledge, there is no simulation software solution available for testing shape sensors. In [13] one first solution is presented, that enables the generation of pseudo-data for shape sensors with parallel guided fibers. By giving the sensor configuration and the fiber position and also one state of deformation, the wavelength values that theoretically are supposed to be measured, are calculated. Fig. 2 shows the graphical user interface of that simulation tool. Although this tool works in case of the standard way of fiber guiding, one more general solution is required that satisfies the requirements, necessary for targeted optimization and testing of shape sensors. As the general design of shape sensors is planned to be modified, a tool that simulates data for arbitrarily distributed fibers is needed.

In the following, a new advanced solution is introduced. Therefore, a software is implemented. Using that software tool, pseudo measurement data can be generated for arbi- trary sensor configurations and state of senor deformation. This means in particular that the fibers embedded into the sensor carrier body no longer necessarily have to be guided in parallel along the sensor axis. For the verification of the presented method of pseudo data generation, the software is tested on its main functionality and on its intuitive and easy usability.

The software tool is divided into three parts. First the target sensor deformation can be entered, secondly the sensor configuration has to be defined. In the last step, the input values are used to calculate the pseudo measurement data. All parts are distinguished through the underlying algorithms, specially adapted to the application, leading to an extended range of use cases and increasing the authenticity of the pseudo-data.

In 2, the specific requirements and characteristics of pseudo data for shape sensing are identified. The implementation of the pseudo data generation software is presented in 3 and evaluated in 4. Finally, in 5, the results are discussed and the remaining work for the future is pointed out.

\section{Pseudo-Measurement Data for Shape Sensing}

Pseudo-data is defined as "(computing) data that is artificially generated in order to test a program [...]"[14]. Regarding shape sensing, the generated pseudo data is supposed to be used especially for testing new algorithms and new sensor configurations. This way, basic functionalities can be evaluated, saving significant material and time cost for building sensor prototypes. Pseudo data is generated from theoretical and physical considerations and should not to be confused with synthetic data, that is produced based on previously measured real data in purpose to anonymise the required data.

Given these objectives, creating pseudo-measurement data requires some basic consideration.

First, it must be specified which kind of data is required, representing the sensor measurement data. Second, the input values, describing the sensor configuration and state of deformation, have to be determined. Finally, a way of calculating the pseudo-data from these input values has to be found.

To specify the kind of data that has to be produced, real data has to be observed. The FBG-Sensors, used for the regarded shape sensing system, measure the wavelength of light. Each embedded fiber returns one wavelength value per inscribed grating. This value varies with stretching or compressing the fiber and therefor stand for the state of strain at the material at discrete points along the sensor. The deformation value $\Delta l$ can be calculated by means of a formula, that describes a linear relationship between the wavelength $\lambda$ ( $\lambda_{B}$ : wavelength, measured in the unloaded condition, $\Delta \lambda_{B}$ : change in wavelength, due to sensor deformation) and the deformation $\Delta l$ as in Eq. 1:

$$
\Delta \lambda_{B}=k \Delta l \lambda_{B}
$$

In this regard, the simulation software output can either be wavelengths or strain values. As strain values are needed 
for shape reconstruction algorithms, the presented software calculates those as output values.

It is now necessary to know, what input values have to be provided for generating pseudo data. Instead of having a real sensor in a specific state of deformation, the possible configuration and deformation state have to be simulated. The configuration is mainly given by the size of the sensor and the positioning of the embedded fibers. As the sensor has a cylindrical form, the sensor size is given by one parameter standing for the sensor length $S$ and by one, representing the diameter $d$ of the cross section. The positioning of the fibers can be defined by the position and orientation of the gratings belonging to it. This means there are $n$ parameter sets, each describing the placement of one grating, with a total number of $n$ gratings. One aim of the software presented in this paper, is that all kinds of sensor configurations should be definable. This leads to a set of five parameters per grating, listed in Tab. 1 and illustrated in Fig. 3:

\begin{tabular}{|l|l|}
\hline Parameter & Description \\
\hline \hline $\mathrm{s}$ & Position along the sensor axis \\
\hline $\mathrm{r}$ & Distance to the sensor axis \\
\hline$\varphi$ & $\begin{array}{l}\text { Direction of the position around } \\
\text { the sensor axis }\end{array}$ \\
\hline$\alpha$ & $\begin{array}{l}\text { Orientation of the grating towards } \\
\text { the sensor axis }\end{array}$ \\
\hline$\beta$ & $\begin{array}{l}\text { Orientation of the grating towards } \\
\text { the tangent vector }\end{array}$ \\
\hline
\end{tabular}

Table 1: Parameters, defining the sensor configuration

Together, this makes a number of three plus five times $n$ parameters that have to be set for a complete definition of the sensor configuration.

To simulate the state of sensor deformation, an arbitrary shape of the sensor axis has to be indicated. The sensor shape can than be derived unambiguously from that axis shape. The axis has to be defined as a space curve via a function in parametric form, where the length of the curve has to correspond to the length $S$ of the sensor.

Finally, it has to be determined how the strain values can be derived from the given input information. To get the measu-

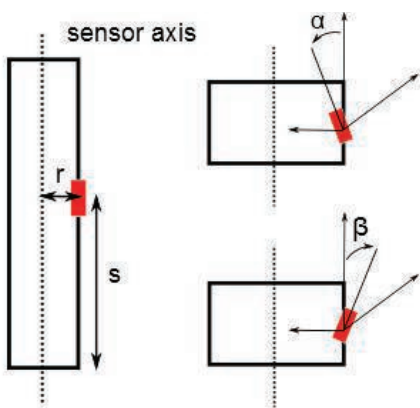

Front view

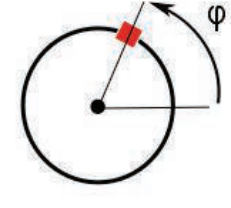

Top view
Abbildung 3 Parameters, defining the sensor configuration rement values, the change in length of the gratings caused by sensor deformation has to be calculated. Therefore, each grating is mapped onto the deformed sensor shape and the new grating length is compared with the grating length in the straight sensor state, which gives the demanded strain value. This calculation is based on geometrical and physical assumptions. To map the grating to the new sensor shape, the so called Frenet-Frame moving along the given axis shape, is defined. This frame consists of three vector functions, whereas one always points in the direction of the curvature.

The generated data represent the theoretically expected measurement values without noise. To produce more realistic data, additional noise has to be simulated. Currently, the focus of research lies in verifying the main functionality of new algorithms. Therefore, no simulated noise is required. Data affected by simulated noise can be used to test the resilience and robustness of the sensor system and is planned for the future.

The described generation process of pseudo-data is realized in a software tool, implemented at the IAR-IPR-KIT, presented in the following chapter.

\section{Implementation of a Pseudo-Data Software Tool}

Based on the considerations of 2, a software tool is developed, that meets certain additional requirements concerning the usability and expandability of the software. Therefore, the software is designed in a manner, which it is easy and intuitive to use and enables to produce pseudo-data in a short period of time. The program was implemented based on a previously defined criteria catalog including fundamental principles of intuitive use to ensure the ease-of-use of the program. Furthermore, the software is programmed in a modular way with open interfaces to ensure the possibility of extending the application and of using the functions for automated sensor optimization based on theoretical data or software in the loop simulation in the future.

The tool consists of thee parts. First, the input data has to

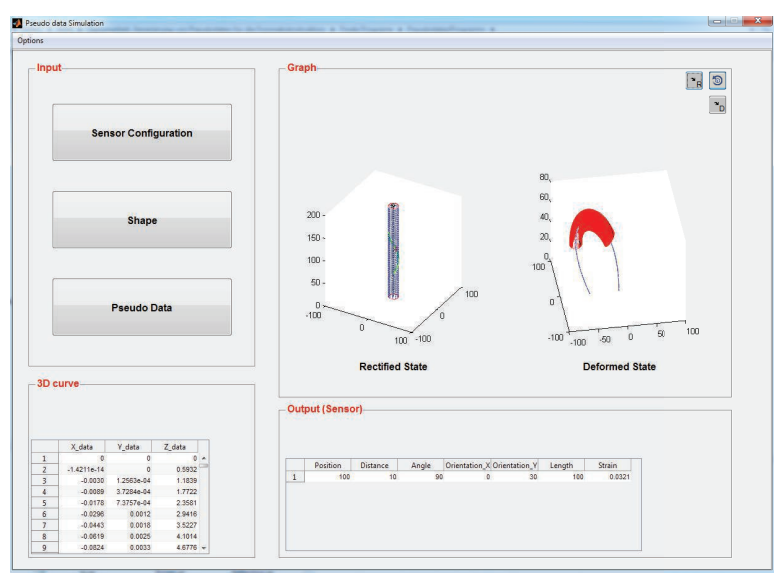

Abbildung 4 Main menu of the software tool 


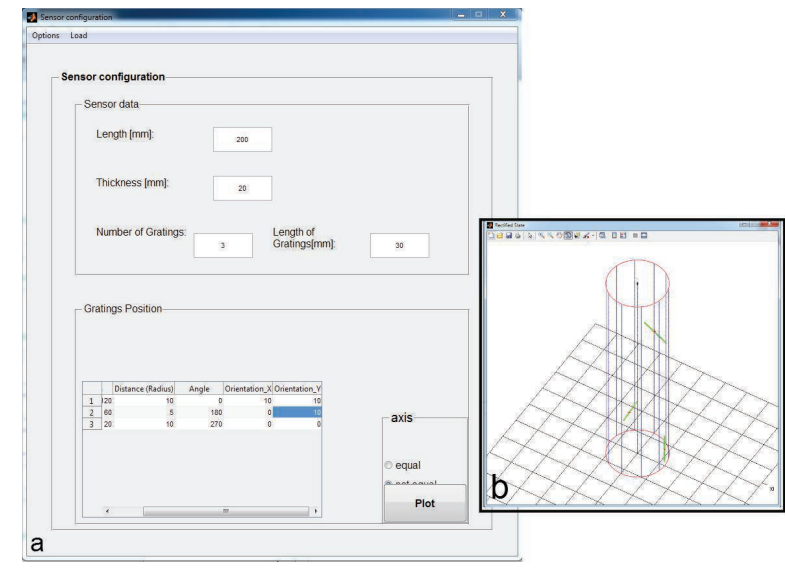

Abbildung 5 Sensor configuration: a - Input window, b Plot of the determined configuration

be entered. These settings are divided into two parts that can be performed independently and in any order. In one part, the sensor configuration can be selected. In the other one, the deformation can be defined. The third part finally calculates the pseudo-data based on the considerations presented in 2 and can only be called after finishing the input settings. Fig. 4 shows the graphical user interface of the software start menu.

To ensure intuitive usability, all icons are clearly arranged and a help function is provided, that calls up a handbook with explanations of all functions of the program.

Selecting the Design option, the configuration of the sensor can be entered (See Fig. 5 a).

After setting the sensor length, diameter and number of integrated gratings, the grating specific parameters can be entered per grating. All settings can be entered manually or loaded from former defined sensor configurations or excelfiles. If all parameters are entered, the corresponding sensor design can be plotted (See Fig. 5 b). The unique feature of this software part compared to former tools is the the increased flexibility in defining the sensor properties as not only parallel fiber guiding but all possible ways of grating positioning can be inserted.

Selecting the shape option, the axis shape can be defined (See Fig. 6 a).

There are several possible implemented options that can be chosen to enter the axis shape in 3D space. These options can be classified into two subgroups. On the one hand, there are parametric curves, that can be entered by a discrete

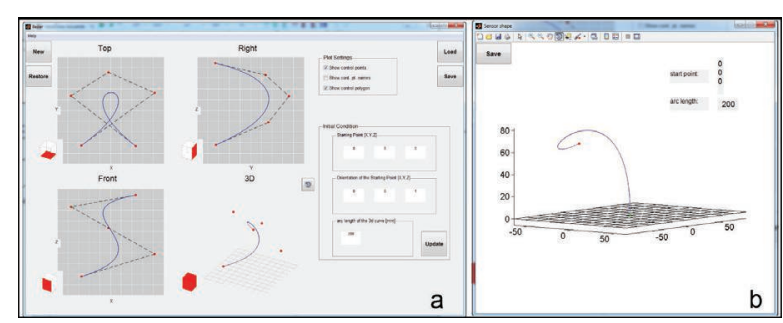

Abbildung 6 Axis shape deformation: a - Input window, $\mathrm{b}$ - Plot of the determined sensor axis shape

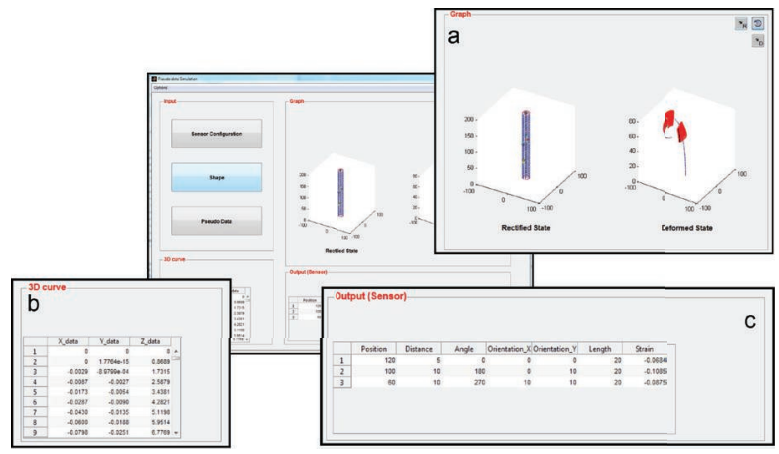

Abbildung 7 Output window: a - Sensor configuration and deformation plot, $\mathrm{b}$ - Coordinate values, describing the sensor shape, $\mathrm{c}$ - Parameter values of the sensor configuration and resulting pseudo measurement data

number of control points, that are interpolated via a Bézier curve, a B-Spline curve or a cubic spline interpolation. On the other hand, it is possible to enter freehand curves using the mouse, the cursor or a drawing tablet. The curves entered this way are smoothed in a second editing step to an individually definable degree. For both types of curve entering, the axis can be entered and adjusted in 3 views (top, front and side view). The input interface is shown in Fig. 6 b. This way of entering the axis shape allows significantly more flexibility in defining different $3 \mathrm{D}$ shapes in an intuitive way. By comparison, as entering options in former programs as e.g. the program presented in [13], it was only possible to define the axis progression by analytic functions in mathematical exact form.

After entering the sensor configuration and the axis shape, in the last program part it is now possible to generate the pseudo-measurement data. The data is saved in an excelfile together with all corresponding information about the input values (shape and configuration) entered in the first two program parts. The data represents the measurement values as strain values and is calculated as explained in 2 . The output program window is shown in Fig. 7.

\section{Evaluation of the Software-Tool}

In the framework of the evaluation, two aspects are considered. First, the functionality of the software and the plausibility of the generated data is observed. Second, the intuitive and easy usability are examined.

Regarding the functionality of the software, for the time being, only theoretical considerations and tests have been carried out. Therefore, all different program parts have been systematically executed to identify and eliminate bugs. Also, the plausibility of the produced data has been observed and compared considering fundamental geometry based on a sample basis. To test the plausibility of the data in a well-founded procedure, test series in which real measurement data will be compared to pseudo data are planed for the near future.

The intuitive usability of the program has been evaluated by means of a user study. The study participants had to per- 
form tasks using the software and thereafter fill out a questionnaire. The study included 30 participants aged between 20 and 35 years and with average or above-average technical know-how. The survey included one testing sequence, in which program parts had to be tested separately and another final sequence in which the whole generation progress was evaluated.

The evaluation points out, that all tasks were at least partly solved. The greatest difficulty is to get used to the way of entering the axis shape. This program part requires some practice but becomes easy to handle after some use. The help function proves to be very useful at this part of the generation process. The combination of the individual stages to complete one entire generation process of pseudo-data was easy to understand and to perform.

This was tested by giving input values for the sensor configuration (See Fig. 8 a) and a picture of a deformed endoscope (See Fig. 8 b) .

$30 \%$ of the participants rate their performance as satisfactory and $55 \%$ as partly sucsessfull. Only the remaining $15 \%$ have not been satisfied with the results.

Responding to the survey, some adjustments of the software have already been made as e.g. the icon arrangement has been modified and the help function has been extended. Further development of the program is planned to be constantly continued.

\section{$5 \quad$ Discussion and Future Work}

Regarding the presented work, the introduced software tool provides a comprehensive and well founded solution, satisfying the requirements demanded. It gives a solid basis for simulative sensor tests and further optimization of the sensor algorithms and configuration. The tool facilitates efficient and targeted research. One main advantage is, that an arbitrary high number of different sensor types can be tested without any upcoming cost of material or time for building prototypes.

At the IAR-IPR-KIT, the software is already in use in some cases and is planned to be integrated into the development process.

The intuitive use of the software is already sufficiently well advanced. The plausibility of the generated data will be tested by comparing the pseudo data with real measured data. A test series is planned to be carried out as a next step.

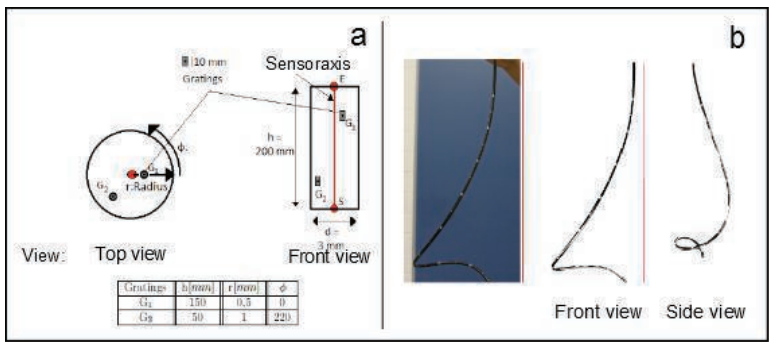

Abbildung 8 Evaluation: a - Given sensor configuration, b - Given shape
Basically, the software is already working well. However, noise is not considered in any way. To test e.g. the robustness of the sensor system, artificial noise has to be produced. Since the software is only used to test the main functionality of new algorithms or new arrangements of the grating positioning, noise can be neglected for now.

Further future work will be the integration of the software into different applications. First, the sensor calibration can be brought to another level. Instead of only calibrating the sensor in straight state to get start values for the algorithm, the algorithm itself can be adapted by adjusting the parameters to the present design.

Another potential application is the use of the software for automated sensor design optimization, that calculates the best way of fiber guiding based on the number of integrated gratings and the deformation character of the used carrier body material.

To conclude, it can be said that one advanced and standing solution has successfully been implemented that provides a good base on which further developments can be built.

\section{$6 \quad$ Literatur}

[1] J. P. Moore and M. D. Rogge, "Shape sensing using multi-core fiber optic cable and parametric curve solutions," in Optics Express, Vol. 20, Issue 3, 2012, pp. 2967-2973.

[2] J. Arkwright, S. Doe, N. Blenman, I. Underhill, S. Maunder, B. Lim, M. Szczesniak, P. Dinning, and I. Cook, "The use of wavelength division multiplexed fiber bragg grating sensors for distributed sensing of pressure in the gastrointestinal tract," in PhotonicsGlobal@Singapore, 2008. IPGC 2008. IEEE, Dec 2008, pp. 1-4.

[3] Z. Lunwei, Q. Jinwu, S. Linyong, and Z. Yanan, "Fbg sensor devices for spatial shape detection of intelligent colonoscope," in Robotics and Automation, 2004. Proceedings. ICRA '04. 2004 IEEE International Conference on, vol. 1, April 2004, pp. 834-840 Vol.1.

[4] Y.-L. Park, S. Elayaperumal, B. Daniel, S. C. Ryu, M. Shin, J. Savall, R. Black, B. Moslehi, and M. Cutkosky, "Real-time estimation of 3-d needle shape and deflection for mri-guided interventions," Mechatronics, IEEE/ASME Transactions on, vol. 15, no. 6, pp. 906-915, Dec 2010.

[5] X. Yi, J. Qian, Y. Zhang, Z. Zhang, and L. Shen, "3d shape display of intelligent colonoscope based on fbg sensor array and binocular vision," in Complex Medical Engineering, 2007. CME 2007. IEEE/ICME International Conference on, May 2007, pp. 14-19.

[6] L. Zhang, J. Qian, Y. Zhang, and L. Shen, "On $\mathrm{sdm} / \mathrm{wdm}$ fbg sensor net for shape detection of endoscope," in Mechatronics and Automation, 2005 IEEE International Conference, vol. 4, July 2005, pp. 1986-1991 Vol. 4.

[7] L.-Y. Shao, L. Xiong, C. Chen, A. Laronche, and 
J. Albert, "Directional bend sensor based on re-grown tilted fiber bragg grating," Lightwave Technology, Journal of, vol. 28, no. 18, pp. 2681-2687, Sept 2010.

[8] X. Chen, C. Zhang, D. Webb, K. Kalli, and G.-D. Peng, "Highly sensitive bend sensor based on bragg grating in eccentric core polymer fiber," Photonics Technology Letters, IEEE, vol. 22, no. 11, pp. 850852, June 2010.

[9] H. Pauer, C. Ledermann, O. Weede, and H. Woern, "Towards building a miniaturized shape sensor: Building process of a shape sensor for use in single port surgery," in Sensing Technology (ICST), 2013 Seventh International Conference on, Dec 2013, pp. 549-554.

[10] C. Ledermann, H. Pauer, H. Woern, M. Seyfried, G. Domann, and H. Wolter, "Using ormocer s as casting material for a $3 \mathrm{~d}$ shape sensor based on fiber bragg gratings," in Intelligent Sensors, Sensor Networks and Information Processing (ISSNIP), 2014 IEEE Ninth International Conference on, April 2014, pp. 1-6.

[11] H. Pauer, C. Ledermann, and H. Woern, "Motivation of a new approach for shape reconstruction based on fbg-optical fibers: Considering of the bragg-gratings composition as a sensornetwork," in Intelligent Sensors, Sensor Networks and Information Processing (ISSNIP), 2014 IEEE Ninth International Conference on, April 2014, pp. 1-5.

[12] H. Pauer, C. Ledermann, W. Tuschmann, and H. Woern, "Non-linear compensation of production inaccuracies and material drift by adjusting the sensor data fusion algorithms for shape sensing based on fbg-optical fibers," in Multisensor Fusion and Information Integration for Intelligent Systems (MFI), 2014 International Conference on, Sept 2014, pp. 15.

[13] C. Ledermann, H. Pauer, O. Weede, and H. Woern, "Simulation tool for $3 \mathrm{~d}$ shape sensors based on fiber bragg gratings and optimization of measurement points," in Robotics, Automation and Mechatronics (RAM), 2013 6th IEEE Conference on, Nov 2013, pp. 195-200.

[14] pseudodata. [Online]. Available: http://www.yourdictionary.com/pseudodata 